\title{
COEXISTENCE OF NON-CONVENTIONAL CONDENSATES IN TWO-LEVEL BOSE ATOM SYSTEM
}

\author{
M. CORGINI ${ }^{1}$, C. ROJAS-MOLINA ${ }^{2}$, AND D.P. SANKOVICH ${ }^{3}$
}

\begin{abstract}
In the framework of the Bogolyubov approximation and using the Bogolyubov inequalities we give a simple proof of the coexistence of two nonconventional Bose-Einstein condensates in the case of some superstable Bose system whose atoms have an internal two-level structure and their energy operators in the second quantized form depend on the number operators only.
\end{abstract}

\section{INTRODUCTION}

The phenomenon of Bose-Einstein condensation (BEC) was described first by Einstein [1]. Until recently the best experimental evidence that BEC could occur in a real physical system was the phenomenon of superfluidity in liquid helium as suggested originally by London who introduced the concept of macroscopic occupation of the ground state [2] and conjectured that the momentum-space condensation of bosons is enhanced by spatial repulsion between the particles [3]. However, nowadays, there exists a considerable amount of experimental evidence for BEC [4, 5].

Given the difficulty of the problem of proving the existence of BEC from a mathematical point of view, it is desirable to have idealized models in which one can develop concrete scenarios for BEC. In this sense, we are interested in studying Bose systems whose energy operators consider repulsive mean interactions represented by diagonal operators in the occupation numbers. It frequently leads to thermodynamically stable systems which can be classically understood.

We are considering a class of systems for which various Bose condensates, in the sense of macroscopic occupation of the ground state, coexist [6, 7]. Specifically we are interested in the study of thermodynamic behavior of some systems for which the Bose atoms have an internal two-level structure. We prove that the existence of two ground state levels, one of them with negative energy, leads to an enhancement of condensation.

This paper is divided as follows. In section 2 we describe the basic mathematical notions associated to this kind of systems and we study the stability of a system of atoms with internal structure. In section 3 making use of the so-called Bogolyubov approximation [8, 9] we give an extremely simple proof of non-conventional BEC in the sense of macroscopic occupation of the ground state [10, 11, 12]. We prove that the existence of two ground state levels, one of them with negative energy, leads 
to an enhancement of condensation in the sense of macroscopic occupation of both internal states [6, 7]. The proposed approximation (Bogolyubov approximation) is obtained by substitution of the zero mode creation and annihilation Bose operators by suitable chosen c-numbers. In this proof a significative role play a well known variational Bogolyubov inequalities and the fact that the involved Hamiltonians are written in terms of the number operators. It enables us to give a simplified demonstration of thermodynamic equivalence of the limit grand canonical pressures corresponding to the energy operator of the system and the respective approximating Hamiltonian.

Atoms BEC's in two different hyperfine states confined in a single trap with a time-varying Raman coupling between the two levels are studied in Ref.13. The Hamiltonian associated to such system, being $\hat{a}, \hat{b}$ the annihilation Bose operators corresponding to the two different states and under the two mode approximation is given as:

$$
\begin{gathered}
\hat{H}=\hat{H}_{a}+\hat{H}_{b}+\hat{H}_{\mathrm{int}}+\hat{H}_{\mathrm{las}}, \\
\hat{H}_{a}=\omega_{a} \hat{a}^{\dagger} \hat{a}+\frac{U_{a}}{2} \hat{a}^{\dagger} \hat{a}^{\dagger} \hat{a} \hat{a}, \\
\hat{H}_{b}=\omega_{b} \hat{b}^{\dagger} \hat{b}+\frac{U_{b}}{2} \hat{b}^{\dagger} \hat{b}^{\dagger} \hat{b} \hat{b}, \\
\hat{H}_{\mathrm{int}}=\frac{U_{a b}}{2} \hat{a}^{\dagger} \hat{a} \hat{b}^{\dagger} \hat{b}, \\
\hat{H}_{\text {las }}=\Omega(t)\left(e^{i \phi t} \hat{a}^{\dagger} \hat{b}+e^{-i \phi t} \hat{b}^{\dagger} \hat{a}\right),
\end{gathered}
$$

where $\hat{H}_{a}, \hat{H}_{b}$ describe systems with self interactions. $\hat{H}_{a b}$ is associated to collisions between both systems and $U_{a}, U_{b}, U_{a b}$ are constants. Finally $\hat{H}_{\text {las }}$ represents the operator associated to the Raman coupling.

We shall prove by using techniques of Bogolyubov approximation that an analogous scenario for the coexistence of different condensates can be obtained in the case of superstable systems of two-level Bose-atoms, described by energy operators of the mean field type, undergoing self interactions and collisions. Obviously the Raman coupling is excluded from the respective Hamiltonians.

\section{The Model}

The one-particle free Hamiltonian corresponds to the operator $S^{l}=-\frac{\Delta}{2}$ defined on a dense subset of the Hilbert space $\mathcal{H}^{l}=L^{2}\left(\Lambda_{l}\right)$, being $\Lambda_{l}=\left[-\frac{l}{2}, \frac{l}{2}\right]^{d} \subset \mathbb{R}^{d}$ a cubic box of boundary $\partial \Lambda_{l}$ and volume $V_{l}=l^{d}$. In other words, the particles are confined to bounded regions. We assume periodic boundary conditions under which $S^{l}$ becomes a self-adjoint operator.

In this section it is assumed that the Bose atoms have an internal two-level structure analogous to the $\mathrm{SU}(2)$ spin symmetry [6, 7]. In this case any one-particle 
wave function has the form $\phi \otimes s$ where, $\phi \in L^{2}\left(\Lambda_{l}\right)$ and $s \in \mathbb{C}^{2}$ represents the internal state. Therefore the vector space associated to this system is in fact, $\mathcal{H}_{s}^{l}=L^{2}\left(\Lambda_{l}\right) \otimes \mathbb{C}^{2}$.

We shall study the model of Bose particles whose Hamiltonian is given as:

$$
\hat{H}_{l}=\hat{H}_{l}^{0}+\hat{U}_{l}^{I}=\hat{H}_{l}^{0}+\frac{\gamma_{0}}{V_{l}} \sum_{\sigma, \mathbf{p} \in \Lambda_{l}^{*}}\left(\hat{a}_{\mathbf{p}, \sigma}^{\dagger}\right)^{2} \hat{a}_{\mathbf{p}, \sigma}^{2}+\frac{\gamma}{V_{l}} \hat{n}_{\mathbf{0},-} \hat{n}_{\mathbf{0},+}+V_{l} g\left(\frac{\hat{N}^{\prime}}{V_{l}}\right),
$$

where $g(x) \geq a x^{2}, a>0$ is a continuous function and $\sigma=+$ or - depending on the corresponding label of internal energy. The second term at the right hand side of eq. (6) represents the intrastate collisions, or the self-scattering, the third term represents the interstate collisions or the cross scattering. The sum in (6) runs over the set $\Lambda_{l}^{*}=\left\{\mathbf{p} \in \mathbb{R}^{d}: p_{\alpha}=2 \pi n_{\alpha} / l, n_{\alpha}=0, \pm 1, \pm 2,, \ldots \alpha=1,2, . . d\right\}$, $\hat{a}_{\mathbf{p}, \sigma}^{\dagger}, \hat{a}_{\mathbf{p}, \sigma}$ are the Bose operators of creation and annihilation of particles defined on the Fock space $\mathcal{F}_{B}$ and satisfying the usual commutation rules: $\left[\hat{a}_{\mathbf{q}, \sigma_{1}}, \hat{a}_{\mathbf{p}, \sigma_{2}}^{\dagger}\right]=$ $\hat{a}_{\mathbf{q}, \sigma_{1}} \hat{a}_{\mathbf{p}, \sigma_{2}}^{\dagger}-\hat{a}_{\mathbf{p}, \sigma_{2}}^{\dagger} \hat{a}_{\mathbf{q}, \sigma_{1}}=\delta_{\mathbf{p}, \mathbf{q}} \delta_{\sigma_{1}, \sigma_{2}} \cdot \hat{n}_{\mathbf{p}, \sigma}=\hat{a}_{\mathbf{p}, \sigma}^{\dagger} \hat{a}_{\mathbf{p}, \sigma}$ is the number operator associated to index $\mathbf{p}$ and internal label $\sigma$ and

$$
\lambda_{l, \sigma}(\mathbf{p})= \begin{cases}\sigma \lambda, & \mathbf{p}=\mathbf{0} \\ \frac{\|\mathbf{p}\|^{2}}{2}, & \mathbf{p} \neq \mathbf{0} .\end{cases}
$$

In this case $\hat{H}_{l}^{0}=\sum_{\sigma, \mathbf{p} \in \Lambda_{l}^{*}} \lambda_{l, \sigma}(\mathbf{p}) \hat{n}_{\mathbf{p}, \sigma}$ and $\gamma_{0}, \gamma>0 . \hat{N}=\sum_{\sigma, \mathbf{p} \in \Lambda_{l}^{*}} \hat{a}_{\mathbf{p}, \sigma}^{\dagger} \hat{a}_{\mathbf{p}, \sigma}$ is the total number operator and $\hat{N}^{\prime}=\sum_{\sigma, \mathbf{p} \in \Lambda_{l}^{*} \backslash\{\mathbf{0}\}} \hat{a}_{\mathbf{p}, \sigma}^{\dagger} \hat{a}_{\mathbf{p}, \sigma}$ is the total number operator with exclusion of $\hat{n}_{\mathbf{0}, \sigma}$.

Note that the boson Fock space $\mathcal{F}_{B}$ is isomorphic to the tensor product $\otimes_{\sigma, \mathbf{p} \in \Lambda_{l}^{*}} \mathcal{F}_{\mathbf{p}, \sigma}^{B}$ where $\mathcal{F}_{\mathbf{p}, \sigma}^{B}$ is the boson Fock space constructed on the one-dimensional Hilbert space $\mathcal{H}_{\mathbf{p}, \sigma}=\left\{\theta e^{i \mathbf{p} \cdot \mathbf{x}} \otimes e_{\sigma}\right\}_{\theta \in \mathbb{C}}$, where $e_{-}=(0,1)$ and $e_{+}=(1,0)$.

Let

$$
p_{l}(\beta, \mu)=\frac{1}{\beta V_{l}} \ln \operatorname{Tr}_{\mathcal{F}_{B}} \exp \left[-\beta\left(\hat{H}_{l}-\mu \hat{N}\right)\right]
$$

be the grand-canonical pressure corresponding to $\hat{H}_{l}$, where $\beta=\theta^{-1}$ is the inverse temperature. If $\hat{H}_{l}(\mu)=\hat{H}_{l}-\mu \hat{N}$, the equilibrium Gibbs state (grand canonical ensemble $)\langle-\rangle_{\hat{H}_{l}(\mu)}$ is defined as

$$
\langle\hat{A}\rangle_{\hat{H}_{l}(\mu)}=\left[\operatorname{Tr}_{\mathcal{F}_{B}} \exp \left(-\beta \hat{H}_{l}(\mu)\right)\right]^{-1} \operatorname{Tr}_{\mathcal{F}_{B}}\left[\hat{A} \exp \left(-\beta \hat{H}_{l}(\mu)\right)\right]
$$

for any operator $\hat{A}$ acting on the symmetric Bose-Fock space. The total density of particles $\rho(\mu)$ for infinite volume is defined as

$$
\lim _{V_{l} \rightarrow \infty}\left\langle\frac{\hat{N}}{V_{l}}\right\rangle_{\hat{H}_{l}(\mu)}=\lim _{V_{l} \rightarrow \infty} \rho_{l}(\mu)=\rho(\mu)
$$


and the density of particles $\rho_{\mathbf{p}, \sigma}(\beta, \mu)$ associated to the energy label $\lambda_{\sigma}(\mathbf{p})$ is defined as

$$
\lim _{V_{l} \rightarrow \infty}\left\langle\frac{\hat{n}_{\mathbf{p}, \sigma}}{V_{l}}\right\rangle_{\hat{H}_{l}(\mu)}=\lim _{V_{l} \rightarrow \infty} \rho_{\mathbf{p}, \sigma, l}(\beta, \mu)=\rho_{\mathbf{p}, \sigma}(\beta, \mu) .
$$

We shall say that the system undergoes a macroscopic occupation of the single $(\mathbf{p}, \sigma)$-mode particle level if $\rho_{\mathbf{p}, \sigma}(\beta, \mu)>0$.

In the case of models with a two label internal structure, [6, 7] two macroscopic occupations independent on temperature (non-conventional BEC) in the sense that

$$
\rho_{\mathbf{0},-}(\mu)>0, \quad \rho_{\mathbf{0},+}(\mu)>0
$$

can coexist.

We are interested in proving the superstability of the model, in other words we verify that the limit pressure $\lim _{V_{l} \rightarrow \infty} p_{l}(\beta, \mu)$ exists for all $\mu \in \mathbb{R}$. A useful criterion [14] says that we are in presence of a superstable system if given an interaction $\hat{U}_{l}^{\mathrm{I}}$ defined on the Bose Fock space $\mathcal{F}_{B}$, there exist two constants $C_{1}>0$ and $C_{2} \geq 0$ such that

$$
\hat{U}_{l}^{\mathrm{I}} \geq-\frac{C_{2}}{V_{l}} \hat{N}+\frac{C_{1}}{V_{l}} \hat{N}^{2}
$$

Then, the superstability of the system whose energy operator has the form (6) is ensured by the following result:

Proposition 2.1. The operator $\hat{U}_{l}^{\mathrm{I}}$ given by (6) satisfies the inequality

$$
\hat{U}_{l}^{\mathrm{I}} \geq-\frac{\gamma_{0}}{V_{l}} \hat{N}+\frac{\min \left\{a, \gamma_{0}\right\}}{4 V_{l}} \hat{N}^{2}
$$

Proof. For $x, y \in \mathbb{R}$ we have $x^{2}+y^{2} \geq(x+y)^{2} / 2$. Moreover, since $\left(\hat{a}_{\mathbf{p}, \sigma}^{\dagger}\right)^{2}\left(\hat{a}_{\mathbf{p}, \sigma}\right)^{2}=$ $\hat{n}_{\mathbf{p}, \sigma}\left(\hat{n}_{\mathbf{p}, \sigma}-1\right) \geq 0$ and the spectrum $\operatorname{Sp}\left(\hat{n}_{\mathbf{p}, \sigma}\right)=\mathbb{N} \cup\{0\}$, for all $\sigma= \pm, \mathbf{p} \in \Lambda_{l}^{*}$, we obtain, in the sense of operators, the following sequence of inequalities: 


$$
\begin{gathered}
\hat{U}_{l}^{\mathrm{I} \geq} \geq-\frac{\gamma_{0}}{V_{l}} \hat{n}_{\mathbf{0},-}-\frac{\gamma_{0}}{V_{l}} \hat{n}_{\mathbf{0},+}+\frac{\gamma_{0}}{V_{l}} \hat{n}_{\mathbf{0},-}^{2}+\frac{\gamma_{0}}{V_{l}} \hat{n}_{\mathbf{0},+}^{2}+V_{l} g\left(\frac{\hat{N}^{\prime}}{V_{l}}\right) \\
\geq-\frac{\gamma_{0}}{V_{l}} \hat{N}+\frac{\gamma_{0}}{V_{l}} \hat{n}_{\mathbf{0},-}^{2}+\frac{\gamma_{0}}{V_{l}} \hat{n}_{\mathbf{0},+}^{2}+a \frac{\left(\hat{N}^{\prime}\right)^{2}}{V_{l}} \\
\geq-\frac{\gamma_{0}}{V_{l}} \hat{N}+\frac{\gamma_{0}}{V_{l}} \hat{n}_{\mathbf{0},-}^{2}+\frac{\min \left\{a, \gamma_{0}\right\}}{V_{l}}\left[\hat{n}_{\mathbf{0},+}^{2}+\left(\hat{N}^{\prime}\right)^{2}\right] \\
\geq-\frac{\gamma_{0}}{V_{l}} \hat{N}+\frac{\gamma_{0}}{2 V_{l}} \hat{n}_{\mathbf{0},-}^{2}+\frac{\min \left\{a, \gamma_{0}\right\}}{2 V_{l}}\left[\hat{n}_{\mathbf{0},+}+\hat{N}^{\prime}\right]^{2} \\
\geq-\frac{\gamma_{0}}{V_{l}} \hat{N}+\frac{\min \left\{a, \gamma_{0}\right\}}{2 V_{l}}\left[\hat{n}_{\mathbf{0},-}^{2}+\left[\hat{n}_{\mathbf{0},+}+\hat{N}^{\prime}\right]^{2}\right] \\
\geq-\frac{\gamma_{0}}{V_{l}} \hat{N}+\frac{\min \left\{a, \gamma_{0}\right\}}{4 V_{l}}\left[\hat{n}_{\mathbf{0},-}+\hat{n}_{\mathbf{0},+}+\hat{N}^{\prime}\right]^{2} \\
=-\frac{\gamma_{0}}{V_{l}} \hat{N}+\frac{\min \left\{a, \gamma_{0}\right\}}{4 V_{l}} \hat{N}^{2} .
\end{gathered}
$$

This concludes the proof.

\section{Approximating Hamiltonian}

For any complex number $c \in \mathbb{C}$, let consider the coherent vector $\phi_{c, \sigma}$ in $\mathcal{F}_{0, \sigma}$, given as

$$
\phi_{c, \sigma}=e^{-V_{l}|c|^{2} / 2} \sum_{n=0}^{\infty} \frac{1}{n !}\left(V_{l}^{\frac{1}{2}} c\right)^{n}\left(\hat{a}_{\mathbf{0}, \sigma}^{\dagger}\right)^{n} \phi_{\mathbf{0}, \sigma}
$$

where $\phi_{\mathbf{0}, \sigma}=\phi_{\mathbf{0}} \otimes e_{\sigma}$ is the vacuum vector in $\mathcal{F}_{\mathbf{0}, \sigma}$. Then, for any operator $\hat{A}$ defined in $\mathcal{F}_{B}$, it is possible to construct an operator $\hat{A}(c)$ such that

$$
\left\langle\Psi_{1}^{\prime}, \hat{A}(c) \Psi_{2}^{\prime}\right\rangle_{\mathcal{F}_{B}}=\left\langle\Psi_{1}^{\prime} \otimes \phi_{c, \sigma}, \hat{A} \Psi_{2}^{\prime} \otimes \phi_{c, \sigma}\right\rangle_{\mathcal{F}_{B}}
$$

where $\Psi_{1}^{\prime}, \Psi_{2}^{\prime} \in \mathcal{F}_{B}^{\prime}$, being $\mathcal{F}_{B}^{\prime}=\otimes_{\sigma, \mathbf{p} \in \Lambda^{*} \backslash\{\mathbf{0}\}} \mathcal{F}_{\mathbf{p}, \sigma}^{B}$. The transition from operator $\hat{A}$ to operator $\hat{A}(c)$ is known as Bogolyubov approximation and it consists in replacing the operators $\hat{a}_{\mathbf{0}}^{\dagger}$ and $\hat{a}_{\mathbf{0}}$ in any $\hat{A}$ expressed in the normal form ( all creation operators are grouped on the left hand side of the group of annihilation operators in $\hat{A}$ ) operators with the complex numbers $\sqrt{V} \bar{c}$ and $\sqrt{V} c$. Then, in the framework of the Bogolyubov approximation, replacing the operators $\hat{a}_{\mathbf{0}, \sigma}^{\dagger}$ and $\hat{a}_{\mathbf{0}, \sigma}$ in $\hat{H}_{l}(\mu)$ with the complex numbers $\sqrt{V_{l}} \bar{c}, \sqrt{V_{l}} c$, for $\sigma=-$ and $\sqrt{V_{l}} \bar{\eta}, \sqrt{V_{l}} \eta$, for $\sigma=+$, respectively, we get the following approximating Hamiltonian $\hat{H}_{l}^{\text {appr }}(c, \eta, \mu)$, 


$$
\begin{aligned}
\hat{H}_{l}^{\mathrm{appr}}(c, \mu)= & \sum_{\sigma, \mathbf{p} \in \Lambda_{l}^{*} \backslash\{\mathbf{0}\}}\left(\lambda_{l, \sigma}(\mathbf{p})-\mu\right) \hat{n}_{\mathbf{p}, \sigma}+\frac{\gamma_{0}}{V_{l}} \sum_{\sigma, \mathbf{p} \in \Lambda_{l}^{*} \backslash\{\mathbf{0}\}}\left(\hat{a}_{\mathbf{p}, \sigma}^{\dagger}\right)^{2} \hat{a}_{\mathbf{p}, \sigma}^{2} \\
& +V_{l} g\left(\frac{\hat{N}^{\prime}}{V_{l}}\right)+\left(\lambda_{l,-}(\mathbf{0})-\mu\right) V_{l}|c|^{2}+\gamma_{0} V_{l}|c|^{4} \\
& +\left(\lambda_{l,+}(\mathbf{0})-\mu\right) V_{l}|\eta|^{2}+\gamma_{0} V_{l}|\eta|^{4}+\gamma V_{l}|c|^{2}|\eta|^{2}
\end{aligned}
$$

with $c, \eta \in \mathbb{C}$.

Note that $\hat{H}_{l}^{\text {appr }}(c, \mu)$ can be rewritten in a more suggestive form as:

$$
\begin{aligned}
\hat{H}_{l}^{\mathrm{appr}}(c, \mu)=\hat{H}^{\mathrm{MF}^{\prime}}(\mu) & +\frac{\gamma_{0}}{V_{l}} \sum_{\sigma, \mathbf{p} \in \Lambda_{l}^{*} \backslash\{\mathbf{0}\}}\left(\hat{a}_{\mathbf{p}, \sigma}^{\dagger}\right)^{2} \hat{a}_{\mathbf{p}, \sigma}^{2}+\left(\lambda_{l,-}(\mathbf{0})-\mu\right) V_{l}|c|^{2}+\gamma_{0} V_{l}|c|^{4} \\
& +\left(\lambda_{l,+}(\mathbf{0})-\mu\right) V_{l}|\eta|^{2}+\gamma_{0} V_{l}|\eta|^{4}+\gamma V_{l}|c|^{2}|\eta|^{2}
\end{aligned}
$$

where

$$
\hat{H}^{\mathrm{MF}^{\prime}}(\mu) \equiv \sum_{\sigma, \mathbf{p} \in \Lambda_{l}^{*} \backslash\{\mathbf{0}\}}\left(\lambda_{l, \sigma}(\mathbf{p})-\mu\right) \hat{n}_{\mathbf{p}, \sigma}+V_{l} g\left(\frac{\hat{N}^{\prime}}{V_{l}}\right)
$$

denotes the so-called mean field Hamiltonian with excluded zero mode.

In this case we have the following theorem [9].

Theorem 3.1. The following inequality takes place:

$$
\operatorname{Tr}_{\mathcal{F}_{B}^{\prime}} e^{-\beta \hat{H}_{l}^{\mathrm{appr}}(c, \eta, \mu)} \leq \operatorname{Tr}_{\mathcal{F}_{B}} e^{-\beta \hat{H}_{l}(\mu)} .
$$

Proof. From the above definitions it follows that

$$
\begin{gathered}
\operatorname{Tr}_{\mathcal{F}_{B}^{\prime}}\left(e^{-\beta \hat{H}_{l}(c, \eta, \mu)}\right)=\sup _{\left\{\Psi_{n}^{\prime}\right\}} \sum_{n} \exp \left(-\beta\left\langle\Psi_{n}^{\prime}, \hat{H}_{l}(c, \eta, \mu) \Psi_{n}^{\prime}\right\rangle\right) \\
=\sup _{\left\{\Psi_{n}^{\prime}\right\}} \sum_{n} \exp \left(-\beta\left\langle\Psi_{n}^{\prime} \otimes \phi_{c,-} \otimes \phi_{\eta,-}, \hat{H}_{l}(\mu) \Psi_{n}^{\prime} \otimes \phi_{c,-} \otimes \phi_{\eta,+}\right\rangle\right) \\
\leq \sup _{\left\{\Psi_{n}^{\prime}\right\}} \sum_{n}\left\langle\Psi_{n}^{\prime} \otimes \phi_{c,-} \otimes \phi_{\eta,+}, e^{-\beta \hat{H}_{l}(\mu)} \Psi_{n}^{\prime} \otimes \phi_{c,-} \otimes \phi_{\eta,+}\right\rangle \\
\leq \operatorname{Tr}_{\mathcal{F}_{B}}\left(e^{-\beta \hat{H}_{l}(\mu)}\right) .
\end{gathered}
$$

The supremum is over all orthonormal basis of the corresponding Fock space $\mathcal{F}_{B}^{\prime}$. The first inequality is the Peierls inequality; the second one is obtained taking the coherent vector $\phi_{c,-} \otimes \phi_{\eta,+}$ as one of the vectors in the orthonormal basis of $\mathcal{F}_{\mathbf{0}}=\mathcal{F}_{\mathbf{0},-} \otimes \mathcal{F}_{\mathbf{0},+}$. This complets the proof. 
3.1. Bogolyubov Inequalities. Let $\hat{H}_{a, l}$ and $\hat{H}_{b, l}$ be self-adjoined operators defined on $\mathcal{D} \subset \mathcal{F}_{B} . \quad p_{a, l}(\beta, \mu), p_{b, l}(\beta, \mu)$ represent the grand canonical pressures corresponding to the operators $\hat{H}_{a, l}, \hat{H}_{b, l}$. In this case the following well known Bogolyubov inequalities

$$
\begin{aligned}
&\left\langle\frac{\hat{H}_{a, l}(\mu)-\hat{H}_{b, l}(\mu)}{V_{l}}\right\rangle_{\hat{H}_{a, l}(\mu)} \leq p_{b, l}(\beta, \mu)-p_{a, l}(\beta, \mu) \\
& \leq\left\langle\frac{\hat{H}_{a, l}(\mu)-\hat{H}_{b, l}(\mu)}{V_{l}}\right\rangle_{\hat{H}_{b, l}(\mu)},
\end{aligned}
$$

hold, where $\langle-\rangle_{\hat{H}_{a, l}(\mu)},\langle-\rangle_{\hat{H}_{b, l}(\mu)}$ are the Gibbs states in the grand canonical ensemble associated to the Hamiltonians $\hat{H}_{a, l}, \hat{H}_{b, l}$, respectively.

\subsection{Global and Local Minimums for Convex Functions.}

Proposition 3.2. Let $G: U \rightarrow \mathbb{R}$ be convex on a convex set $U \subset L$ ( $L$ is a normed linear space). If $G$ has a local minimum at $\bar{x}$, then $G(\bar{x})$ is also a global minimizer. The set $V$ ( conceivably empty) on which $G$ attains its minimum is convex. And if $G$ is strictly convex in a neighbourhood of a minimum point $\hat{x}$, then $V=\{\hat{x}\}$; that is, the minimum is unique.

The proof is contained in Ref.15.

\subsection{Equivalence of Limit Grand Canonical Pressures.}

Theorem 3.3. Under any of the conditions

(i) $2 \gamma_{0}>\gamma, \mu \geq \lambda\left(2 \gamma_{0}+\gamma\right) /\left(2 \gamma_{0}-\gamma\right)$,

(ii) $\gamma=0, \mu \in \mathbb{R}$,

(iii) $2 \gamma_{0}=\gamma, \mu \in \mathbb{R}$,

the systems with Hamiltonians $\hat{H}_{l}(\mu)$ and $\hat{H}_{l}^{\text {appr }}(c, \eta, \mu)$ are thermodynamically equivalent in the following sense,

$$
\lim _{V_{l} \rightarrow \infty} \sup _{|c|,|\eta|: c, \eta \in \mathbb{C}} p_{l}^{\text {appr }}(\beta, c, \eta, \mu)=p(\beta, \mu),
$$

where $p_{l}^{\text {appr }}(\beta, c, \eta, \mu)=\left(\beta V_{l}\right)^{-1} \ln \operatorname{Tr}_{\mathcal{F}_{B}} \exp \left[-\beta\left(\hat{H}_{l}^{\mathrm{appr}}(c, \eta, \mu)\right)\right]$.

Proof. Applying the Bogolyubov inequalities for pressures (23) and inequality (21), we obtain that:

$$
0 \leq p_{l}(\beta, \mu)-p_{l}^{\mathrm{appr}}(\beta, c, \eta, \mu) \leq \frac{1}{V_{l}}\left\langle\hat{H}_{l}^{\mathrm{appr}}(c, \eta, \mu)-\hat{H}_{l}(\mu)\right\rangle_{\hat{H}_{l}(\mu)} \equiv \Delta_{l}(c, \eta, \mu) .
$$

The right hand side of inequality (25) can be rewritten as, 


$$
\begin{aligned}
\Delta_{l}(c, \eta, \mu)= & \left(\lambda_{l,-}(\mathbf{0})-\mu\right)|c|^{2}+\gamma_{0}|c|^{4}+\left(\lambda_{l,+}(\mathbf{0})-\mu\right)|\eta|^{2}+\gamma_{0}|\eta|^{4}+\gamma|c|^{2}|\eta|^{2} \\
& +\left\langle\left(\sum_{\sigma= \pm}\left(\mu-\lambda_{l, \sigma}(\mathbf{0})\right) \hat{\rho}_{\mathbf{0}, \sigma}-\gamma_{0} \hat{\rho}_{\mathbf{0}, \sigma}\left(\hat{\rho}_{\mathbf{0}, \sigma}-\frac{1}{V_{l}}\right)\right)-\gamma \hat{\rho}_{\mathbf{0},-} \hat{\rho}_{\mathbf{0},+}\right\rangle_{\hat{H}_{l}(\mu)}
\end{aligned}
$$

where $\hat{\rho}_{\mathbf{0}, \sigma}=\hat{n}_{\mathbf{0}, \sigma} / V_{l}$.

(i) Under the condition $2 \gamma_{0}>\gamma$ and according proposition $3.2 g(x, y): \mathbb{R}^{2} \rightarrow \mathbb{R}$ given as:

$$
g(x, y)=\left(\lambda_{l,-}(\mathbf{0})-\mu\right) x+\gamma_{0} x^{2}+\left(\lambda_{l,+}(\mathbf{0})-\mu\right) y+\gamma_{0} y^{2}+\gamma x y,
$$

is a strictly convex function attaining a global minimum at

$$
\begin{aligned}
& x_{\mathbf{0}}=\frac{\gamma\left(\lambda_{l,+}(\mathbf{0})-\mu\right)-2 \gamma_{0}\left(\lambda_{l,-}(\mathbf{0})-\mu\right)}{4 \gamma_{0}^{2}-\gamma^{2}}, \\
& y_{\mathbf{0}}=\frac{\gamma\left(\lambda_{l,-}(\mathbf{0})-\mu\right)-2 \gamma_{0}\left(\lambda_{l,+}(\mathbf{0})-\mu\right)}{4 \gamma_{0}^{2}-\gamma^{2}},
\end{aligned}
$$

with $(x, y) \in \mathbb{R}^{2}$, since $\partial_{x}^{2} g=2 \gamma_{0}>0$ and $\partial_{x} g \partial_{y} g-\left[\partial_{x y}^{2} g\right]^{2}=4 \gamma_{0}^{2}-\gamma^{2}$. Taking $\lambda_{l,-}(\mathbf{0})=-\lambda$ and $\lambda_{l,+}(\mathbf{0})=\lambda$, we have that $g(x, y)=-(\lambda+\mu) x+\gamma_{0} x^{2}+(\lambda-\mu) y+$ $\gamma_{0} y^{2}+\gamma x y$ and

$$
\begin{gathered}
x_{\mathbf{0}}=\left|c_{\mathbf{0}}\right|^{2}=\frac{\lambda}{2 \gamma_{0}-\gamma}+\frac{\mu}{2 \gamma_{0}+\gamma}, \\
y_{\mathbf{0}}=\left|\eta_{\mathbf{0}}\right|^{2}=-\frac{\lambda}{2 \gamma_{0}-\gamma}+\frac{\mu}{2 \gamma_{0}+\gamma},
\end{gathered}
$$

for $\mu \geq \lambda\left(2 \gamma_{0}+\gamma\right) /\left(2 \gamma_{0}-\gamma\right)$. It is easy to verify that:

$$
g\left(x_{\mathbf{0}}, y_{\mathbf{0}}\right)=-\left(\frac{\lambda^{2}}{2 \gamma_{0}-\gamma}+\frac{\mu^{2}}{2 \gamma_{0}+\gamma}\right)
$$

On the other hand, being $\mu_{l}=\mu+\gamma_{0} / V_{l}$ we have

$$
\left\langle g_{l}^{*}\left(\hat{\rho}_{\mathbf{0},-}, \hat{\rho}_{\mathbf{0},+}\right)\right\rangle_{\hat{H}_{l}(\mu)}=\left\langle\left(\sum_{\sigma= \pm}\left(\lambda_{l, \sigma}(\mathbf{0})-\mu_{l}\right) \hat{\rho}_{\mathbf{0}, \sigma}+\gamma_{0} \hat{\rho}_{\mathbf{0}, \sigma}^{2}\right)+\gamma \hat{\rho}_{\mathbf{0},-} \hat{\rho}_{\mathbf{0},+}\right\rangle_{\hat{H}_{l}(\mu)}
$$

where

$$
g_{l}^{*}(x, y)=\left(\lambda_{l,-}(\mathbf{0})-\mu_{l}\right) x+\gamma_{0} x^{2}+\left(\lambda_{l,+}(\mathbf{0})-\mu_{l}\right) y+\gamma_{0} y^{2}+\gamma x y .
$$

With $\lambda_{l,-}(\mathbf{0})=-\lambda, \lambda_{l,+}(\mathbf{0})=\lambda$, and under the same before established conditions $g_{l}^{*}$ has a global minimum at the positive real values

$$
\begin{gathered}
x_{\mathbf{0}, l}^{*}=\frac{\lambda}{2 \gamma_{0}-\gamma}+\frac{\mu_{l}}{2 \gamma_{0}+\gamma}, \\
y_{\mathbf{0}, l}^{*}=-\frac{\lambda}{2 \gamma_{0}-\gamma}+\frac{\mu_{l}}{2 \gamma_{0}+\gamma} .
\end{gathered}
$$


Clearly $x_{\mathbf{0}, l}^{*} \rightarrow x_{\mathbf{0}}$ and $y_{\mathbf{0}, l}^{*} \rightarrow y_{\mathbf{0}}$ when $V_{l} \rightarrow \infty$ and $\lim _{V_{l} \rightarrow \infty} g_{l}^{*}\left(x_{\mathbf{0}, l}^{*}, y_{\mathbf{0}, l}^{*}\right)=g\left(x_{\mathbf{0}}, y_{\mathbf{0}}\right)$. Moreover, $g_{l}^{*}(x, y) \leq g(x, y)$ for all $(x, y) \in \mathbb{R}^{+2}$, then

$$
\inf _{(x, y) \in \mathbb{R}^{+2}} g_{l}^{*}(x, y) \leq \inf _{(x, y) \in \mathbb{R}^{+2}} g(x, y)
$$

which implies that

$$
\inf _{(x, y) \in \mathbb{R}^{+2}} g(x, y)+\sup _{(x, y) \in \mathbb{R}^{+2}}\left\{-g_{l}^{*}(x, y)\right\} \geq 0 .
$$

With these definitions we can rewrite $\Delta_{l}(c, \eta, \mu)$ as,

$$
\Delta_{l}(c, \eta, \mu)=g\left(|\eta|^{2},|c|^{2}\right)+\left\langle-g_{l}^{*}\left(\hat{\rho}_{\mathbf{0},-}, \hat{\rho}_{\mathbf{0},+}\right)\right\rangle_{\hat{H}_{l}(\mu)} .
$$

The energy operator (6) is diagonal respect to the number operators. Therefore, since the spectra of these operators coincide with the set of non negative integers, this model can be classically understood by using non negative random variables defined on a suitable probability space $\Omega_{l}$. Let $\Omega_{l}$ be the countable set of sequences $\omega=\left\{\omega(\mathbf{p}, \sigma) \in \mathbb{N}: \sigma= \pm, \mathbf{p} \in \Lambda_{l}^{*}\right\} \subset \mathbb{N} \cup\{0\}$ satisfying

$$
\sum_{\sigma, \mathbf{p} \in \Lambda_{l}^{*}} \omega(\mathbf{p}, \sigma)<\infty
$$

The basic random variables are the occupation numbers $\left\{n_{\mathbf{p}, \sigma}: j=1,2, \ldots, \sigma= \pm\right\}$. They are defined as the functions $n_{\mathbf{p}, \sigma}: \Omega_{l} \rightarrow \mathbb{N}$ given as $n_{\mathbf{p}, \sigma}(\omega)=\omega(\mathbf{p}, \sigma)$ for any $\omega \in \Omega_{l}$. The total number of particles in the configuration $\omega$ is denoted as $N(\omega)$. Then the total number, excluded the zero mode is denoted as $N^{\prime}(\omega)$.

Let $H_{l}(\mu)$ be the function of the random variables $n_{\mathbf{p}, \sigma}$ defined as

$$
H_{l}(\mu)=\left(\sum_{\sigma, \mathbf{p} \in \Lambda_{l}^{*}}\left(\lambda_{l, \sigma}(\mathbf{p})-\mu_{l}\right) n_{\mathbf{p}, \sigma}+\frac{\gamma_{0}}{V_{l}} n_{\mathbf{p}, \sigma}^{2}\right)+\frac{\gamma}{V_{l}} n_{\mathbf{0},-} n_{\mathbf{0},+}+V_{l} g\left(\frac{N^{\prime}}{V_{l}}\right) .
$$

Let $\mathbb{P}$ be a probability density defined for any $\omega \in \Omega_{l}$ as

$$
\mathbb{P}[\omega]=\left[\sum_{\omega \in \Omega} \exp \left(-\beta\left[H_{l}(\mu)\right](\omega)\right)\right]^{-1} \exp \left(-\beta\left[H_{l}(\mu)\right](\omega)\right) .
$$

Being $\mathbb{E}$ the expectation related to $\mathbb{P}$, the concavity of $-g_{l}^{*}$ implies that this function has a global maximum, then we obtain,

$$
\left\langle-g_{l}^{*}\left(\hat{\rho}_{\mathbf{0},-}, \hat{\rho}_{\mathbf{0},+}\right)\right\rangle_{\hat{H}_{l}(\mu)}=\mathbb{E}\left(-g_{l}^{*}\left(\rho_{\mathbf{0},-}, \rho_{\mathbf{0},+}\right)\right) \leq \mathbb{E}\left(\sup _{(x, y) \in \mathbb{R}^{+2}}\left\{-g_{l}^{*}(x, y)\right\}\right),
$$


where $\rho_{\mathbf{0}, \sigma}=n_{\mathbf{0}, \sigma} / V_{l}$. From this it follows that

$$
\begin{gathered}
0 \leq p_{l}(\beta, \mu)-\sup _{|c|,|\eta|: c, \eta \in \mathbb{C}} p_{l}^{\text {appr }}(\beta, c, \eta, \mu) \leq \inf _{|c|,|\eta|: c, \eta \in \mathbb{C}} \Delta_{l}(c, \eta, \mu) \\
\leq \inf _{|c|,|\eta|: c, \eta \in \mathbb{C}} g\left(|c|^{2},|\eta|^{2}\right)+\sup _{(x, y) \in \mathbb{R}^{+2}}\left\{-g_{l}^{*}(x, y)\right\} .
\end{gathered}
$$

Therefore,

$$
0 \leq \lim _{V_{l} \rightarrow \infty|c|,|\eta|: c, \eta \in \mathbb{C}} \inf _{l}(c, \eta, \mu) \leq g\left(x_{0}, y_{0}\right)+\lim _{V_{l} \rightarrow \infty}\left\{-g_{l}^{*}\left(x_{\mathbf{0}, l}^{*}, y_{\mathbf{0}, l}^{*}\right)\right\}=0 .
$$

(ii) We have that:

$$
\begin{array}{r}
\frac{1}{V_{l}}\left\langle\hat{H}_{l}^{\mathrm{appr}}(c, \eta, \mu)-\hat{H}_{l}(\mu)\right\rangle_{\hat{H}_{l}(\mu)}=-(\lambda+\mu)|c|^{2}+\gamma_{0}|c|^{4} \\
+(\lambda-\mu)|\eta|^{2}+\gamma_{0}|\eta|^{4}+\left\langle(\lambda+\mu) \hat{\rho}_{\mathbf{0},-}-\gamma_{0} \hat{\rho}_{\mathbf{0},-}\left(\hat{\rho}_{0,-}-\frac{1}{V_{l}}\right)\right\rangle_{\hat{H}_{l}(\mu)} \\
+\left\langle(\mu-\lambda) \hat{\rho}_{\mathbf{0},+}-\gamma_{0} \hat{\rho}_{\mathbf{0},+}\left(\hat{\rho}_{\mathbf{0},+}-\frac{1}{V_{l}}\right)\right\rangle_{\hat{H}_{l}(\mu)}
\end{array}
$$

Let $\rho_{\mathbf{0}, \sigma}=\left\langle\hat{\rho}_{\mathbf{0}, \sigma}\right\rangle_{\hat{H}_{l}(\mu)}$. From the above result and noting that

$$
\inf _{|c|,|\eta|: c, \eta \in \mathbb{C}}\left\{p_{l}(\beta, \mu)-p_{l}^{\text {appr }}(\beta, c, \eta, \mu)\right\}=p_{l}(\beta, \mu)-\sup _{|c|,|\eta|: c, \eta \in C} p_{l}^{\text {appr }}(\beta, c, \mu)
$$

and $\left\langle\left(\hat{\rho}_{\mathbf{0}, \sigma}\right)^{2}\right\rangle_{\hat{H}_{l}(\mu)} \geq\left(\left\langle\hat{\rho}_{\mathbf{0}, \sigma}\right\rangle_{\hat{H}_{l}(\mu)}\right)^{2}$ it follows that,

$$
\begin{array}{r}
0 \leq p_{l}(\beta, \mu)-\sup _{|c|: c \in \mathbb{C}} p_{l}^{\mathrm{appr}}(\beta, c, \eta, \mu) \leq \inf _{|c|: c \in C}\left\{-(\lambda+\mu)|c|^{2}+\gamma_{0}|c|^{4}\right\}+ \\
+\inf _{|\eta|: \eta \in C}\left\{(\mu-\lambda)|\eta|^{2}+\gamma_{0}|\eta|^{4}\right\}+ \\
+\left(\mu+\lambda+\frac{\gamma_{0}}{V_{l}}\right) \rho_{\mathbf{0},-}-\gamma_{0} \rho_{\mathbf{0},-}^{2}+ \\
+\left(\mu-\lambda+\frac{\gamma_{0}}{V_{l}}\right) \rho_{\mathbf{0},+}-\gamma_{0} \rho_{\mathbf{0},+}^{2}
\end{array}
$$

Note that

$$
\sup _{\rho_{\mathbf{0}, \sigma} \geq 0}\left\{\left(\mu-\sigma \lambda+\frac{\gamma_{0}}{V_{l}}\right) \rho_{\mathbf{0}, \sigma}-\gamma_{0} \rho_{\mathbf{0}, \sigma}^{2}\right\}= \begin{cases}0, & \mu \leq \sigma \lambda-\frac{\gamma_{0}}{V_{l}} \\ \frac{\left(\mu-\sigma \lambda+\frac{\gamma_{0}}{V_{l}}\right)^{2}}{4 \gamma_{0}}, & \mu>\sigma \lambda-\frac{\gamma_{0}}{V_{l}} .\end{cases}
$$

On the other hand

$$
\inf _{x \geq 0}\left\{(\sigma \lambda-\mu) x+\gamma_{0} x^{2}\right\}= \begin{cases}0, & \mu \leq \sigma \lambda \\ \frac{(\mu-\sigma \lambda)^{2}}{4 \gamma_{0}}, & \mu>\sigma \lambda .\end{cases}
$$


The respective maximum and minimum are attained in the following way,

$$
\rho_{\mathbf{0}, \sigma}^{*}=\left\{\begin{array}{ll}
0, & \mu \leq \sigma \lambda-\frac{\gamma_{0}}{V_{l}} \\
\frac{\mu-\sigma \lambda}{2 \gamma_{0}}, & \mu>\sigma \lambda-\frac{\gamma_{0}}{V_{l}},
\end{array} \quad x_{\mathbf{0}, \sigma}= \begin{cases}0, & \mu \leq \sigma \lambda \\
\frac{\mu-\sigma \lambda}{2 \gamma_{0}}, & \mu>\sigma \lambda .\end{cases}\right.
$$

Combining these results we obtain, in the thermodynamic limit, the proof for the case $\gamma=0$.

(iii) In the case $2 \gamma_{0}=\gamma$ we have $g(x, y)=-(\lambda+\mu) x+\gamma_{0}(x+y)^{2}+(\lambda-\mu) y$.

(a) $\mu \in(-\infty,-\lambda]$. In this case $g(x, y) \geq 0$, for all $(x, y) \in\left(\mathbb{R}^{+}\right)^{2}$, then $\inf _{(x, y) \in\left(\mathbb{R}^{+}\right)^{2}}\{g(x, y)\}=g(0,0)=0$, where the minimum is attained at

$$
x_{\mathbf{0}}=0, y_{\mathbf{0}}=0 \text {. }
$$

(b) $\mu \in(-\lambda, \lambda]$. We have,

$$
\begin{gathered}
\inf _{(x, y) \in\left(\mathbb{R}^{+}\right)^{2}}\{g(x, y)\}=\inf _{(x, y) \in\left(\mathbb{R}^{+}\right)^{2}}\left\{-(\lambda+\mu) x+\gamma_{0}(x+y)^{2}+(\lambda-\mu) y\right\} \\
=\inf _{x \in \mathbb{R}^{+}}\{g(x, 0)\}=\inf _{x \in \mathbb{R}^{+}}\left\{-(\lambda+\mu) x+\gamma_{0} x^{2}\right\}=-\frac{(\mu+\lambda)^{2}}{4 \gamma_{0}},
\end{gathered}
$$

where the minimizer is given by,

$$
x_{\mathbf{0}}=\frac{\mu+\lambda}{2 \gamma_{0}}, y_{0}=0 .
$$

(c) $\mu \in(\lambda, \infty)$. Let $r_{1}=\lambda+\mu, r_{2}=\mu-\lambda$. Then,

$$
g(x, y)=-r_{1} x+\gamma_{0}(x+y)^{2}-r_{2} y
$$

In this case,

$$
\inf _{(x, y) \in\left(\mathbb{R}^{+}\right)^{2}}\{g(x, y)\}=-\frac{\lambda \mu}{\gamma_{0}} .
$$

The minimizer is given by,

$$
x_{0}=\frac{\mu}{\gamma_{0}}, y_{0}=0
$$

This ends the proof.

Corollary 3.4. Assume that $\sup _{r \geq 0}\{(\mu-\alpha) r-g(r)\}$ exists. Then,

$$
p(\beta, \mu)= \begin{cases}\frac{\lambda^{2}}{2 \gamma_{0}-\gamma}+\frac{\mu^{2}}{2 \gamma_{0}+\gamma}+p^{\mathrm{MF}^{\prime}}(\beta, \mu), & 2 \gamma_{0}>\gamma>0, \mu \geq \frac{\lambda\left(2 \gamma_{0}+\gamma\right)}{2 \gamma_{0}-\gamma}, \\ h_{1}(\lambda, \mu)+p^{\mathrm{MF}^{\prime}}(\beta, \mu), & \gamma=0, \gamma_{0} \neq 0, \\ h_{2}(\lambda, \mu)+p^{\mathrm{MF}^{\prime}}(\beta, \mu), & 2 \gamma_{0}=\gamma,\end{cases}
$$


where,

$$
h_{1}(\lambda, \mu)=\left\{\begin{array}{ll}
0, & \mu \in(-\infty,-\lambda] \\
\frac{(\mu+\lambda)^{2}}{4 \gamma_{0}}, & \mu \in(-\lambda, \lambda] \\
\frac{\mu^{2}+\lambda^{2}}{2 \gamma_{0}}, & \mu \in(\lambda, \infty)
\end{array} \quad h_{2}(\lambda, \mu)= \begin{cases}0, & \mu \in(-\infty,-\lambda] \\
\frac{(\mu+\lambda)^{2}}{4 \gamma_{0}}, & \mu \in(-\lambda, \lambda] \\
\frac{\lambda \mu}{\gamma_{0}}, & \mu \in(\lambda, \infty) .\end{cases}\right.
$$

and

$$
p^{\mathrm{MF}^{\prime}}(\beta, \mu)=\sup _{r \geq 0} \inf _{\alpha \leq 0}\left\{(\mu-\alpha) r-g(r)+p^{i d^{\prime}}(\beta, \alpha)\right\}
$$

being $p^{\mathrm{id}^{\prime}}(\beta, \alpha)$ the limit grand canonical pressure for the ideal Bose gas with excluded zero mode at chemical potential $\alpha \leq 0$.

Proof. Let $\hat{H}^{\prime}(\mu)=\hat{H}^{\mathrm{MF}^{\prime}}(\mu)+\frac{\gamma_{0}}{V_{l}} \sum_{\sigma, \mathbf{p} \in \Lambda_{l}^{*} \backslash\{0\}}\left(\hat{a}_{\mathbf{p}, \sigma}^{\dagger}\right)^{2} \hat{a}_{\mathbf{p}, \sigma}^{2}=\hat{H}^{\mathrm{MF}^{\prime}}(\mu)+\frac{\gamma_{0}}{V_{l}} \hat{D}$ and $\hat{H}_{l}^{0^{\prime}}=$ $\sum_{\sigma, p \in \Lambda_{l}^{*} \backslash\{\mathbf{0}\}} \lambda_{l, \sigma}(\mathbf{p}) \hat{a}_{\mathbf{p}, \sigma}^{\dagger} \hat{a}_{\mathbf{p}, \sigma}$. Let $f_{l}^{\Gamma}(\varrho)$ be the canonical energy at finite volume $V_{l}$ and density $\varrho$ associated with the Hamiltonian $\Gamma$, defined as,

$$
f_{l}^{\Gamma}(\varrho)=-\frac{1}{V_{l}} \ln \operatorname{Tr}_{\mathcal{H}_{l}^{(\mathrm{B}, N)}} e^{-\left.\beta \Gamma\right|_{\mathcal{H}_{l}^{(\mathrm{B}, N)}}},
$$

where $\mathcal{H}_{l}^{(\mathrm{B}, N)}$ is the Hilbert space representing a $N$-particles Bose system. Let $\varrho_{0}$ the density of particles in the zero mode. Let $f_{l}^{\prime}\left(\varrho-\varrho_{0}\right), f_{l}^{\mathrm{MF}^{\prime}}\left(\varrho-\varrho_{0}\right), f_{l}^{\mathrm{id}^{\prime}}\left(\varrho-\varrho_{\mathbf{0}}\right)$ be the finite canonical energies associated to the operators $\hat{H}^{\prime}, \hat{H}^{\mathrm{MF}^{\prime}}, \hat{H}_{l}^{0^{\prime}}$ respectively and $f^{\prime}\left(\varrho-\varrho_{0}\right), f^{\mathrm{MF}^{\prime}}\left(\varrho-\varrho_{\mathbf{0}}\right), f^{\mathrm{id}^{\prime}}\left(\varrho-\varrho_{\mathbf{0}}\right)$ their respective limit canonical energies.

We only sketch the proof which can be found in Refs.11,12 in the case $g(x)=a x^{2}$. In this case the following identity can be easily verified:

$$
f_{l}^{\prime}\left(\varrho-\varrho_{\mathbf{0}}\right)=g\left(\varrho-\varrho_{\mathbf{0}}\right)-\frac{1}{\beta V_{l}} \ln \left\langle e^{-\frac{\beta \gamma_{0}}{V_{l}} \hat{D}}\right\rangle_{\hat{H}_{l}^{0^{\prime}}\left(\varrho-\varrho_{\mathbf{0}}\right)}+f_{l}^{\mathrm{id}^{\prime}}\left(\varrho-\varrho_{\mathbf{0}}\right),
$$

where $\langle\cdot\rangle_{\hat{H}_{0, l}\left(\varrho-\varrho_{0}\right)}$, represents the canonical Gibbs state at density $\varrho-\varrho_{\mathbf{0}}$ associated to the ideal gas with the single mode zero excluded. Using the following facts,

$$
\begin{gathered}
\left\langle e^{-\frac{\beta \gamma_{0}}{V_{l}} \hat{D}}\right\rangle_{\hat{H}_{l}^{0^{\prime}}\left(\varrho-\varrho_{0}\right)} \geq e^{-\left\langle\frac{\beta \gamma_{0}}{V_{l}} \hat{D}\right\rangle_{\hat{H}_{l}^{0^{\prime}}}\left(\varrho-\varrho_{\mathbf{0}}\right)}, \\
e^{-\frac{\beta \gamma_{0}}{V_{l}} \hat{D}} \leq 1
\end{gathered}
$$

and noting that

$$
\lim _{V_{l} \rightarrow \infty}\left\langle\frac{\hat{D}}{V_{l}^{2}}\right\rangle_{\hat{H}_{l}^{0^{\prime}}(\varrho-\varrho \mathbf{0})}=0
$$


we get

$$
\lim _{V_{l} \rightarrow \infty} \frac{1}{\beta V_{l}} \ln \left\langle e^{-\frac{\beta \gamma_{0}}{V_{l}} \hat{D}}\right\rangle_{\hat{H}_{l}^{0^{\prime}}\left(\varrho-\varrho_{0}\right)}=0 .
$$

This implies that in the thermodynamic limit

$$
f^{\prime}\left(\varrho-\varrho_{\mathbf{0}}\right)=f^{\mathrm{MF}^{\prime}}\left(\varrho-\varrho_{\mathbf{0}}\right)=g\left(\varrho-\varrho_{\mathbf{0}}\right)+f^{\mathrm{id}}\left(\varrho-\varrho_{\mathbf{0}}\right) .
$$

Then,

$$
\begin{gathered}
p^{\prime}(\beta, \mu)=\sup _{\varrho \geq \varrho_{\mathbf{0}}}\left\{\mu\left(\varrho-\varrho_{\mathbf{0}}\right)-f^{\mathrm{MF}^{\prime}}\right\} \\
=\sup _{\varrho \geq \varrho_{\mathbf{0}}}\left\{\mu\left(\varrho-\varrho_{\mathbf{0}}\right)-g\left(\varrho-\varrho_{\mathbf{0}}\right)-f^{\mathrm{id}^{\prime}}\left(\varrho-\varrho_{\mathbf{0}}\right)\right\},
\end{gathered}
$$

but

$$
f^{\mathrm{id}^{\prime}}\left(\varrho-\varrho_{\mathbf{0}}\right)=\sup _{\alpha \leq 0}\left\{\alpha\left(\varrho-\varrho_{\mathbf{0}}\right)-p^{\mathrm{id}^{\prime}}(\alpha)\right\}
$$

Therefore,

$$
\begin{aligned}
p^{\prime}(\beta, \mu)= & \sup _{\varrho \geq \varrho_{0}} \sup _{\alpha \leq 0}\left\{(\mu-\alpha)\left(\varrho-\varrho_{\mathbf{0}}\right)-g\left(\varrho-\varrho_{\mathbf{0}}\right)+p^{\text {id }^{\prime}}(\alpha)\right\} \\
& =\sup _{r \geq 0} \sup _{\alpha \leq 0}\left\{(\mu-\alpha)(r)-g(r)+p^{\text {id }^{\prime}}(\alpha)\right\} .
\end{aligned}
$$

On the other hand it is clear that in the case $2 \gamma_{0}>\gamma$ we obtain,

$$
\begin{gathered}
p(\beta, \mu)=\sup _{(x, y) \in\left(\mathbb{R}^{+}\right)^{2}}\left\{(\mu+\lambda) x-\gamma_{0} x^{2}+(\mu-\lambda) y-\gamma_{0} y^{2}-\gamma x y\right\}+p^{\prime}(\beta, \mu) \\
=(\mu+\lambda) x_{0}-\gamma_{0} x_{0}^{2}+(\mu-\lambda) y_{0}-\gamma_{0} y_{0}^{2}-\gamma x_{0} y_{0}+p^{\prime}(\beta, \mu) \\
=\left(\frac{\lambda^{2}}{2 \gamma_{0}-\gamma}+\frac{\mu^{2}}{2 \gamma_{0}+\gamma}\right)+p^{\prime}(\beta, \mu) .
\end{gathered}
$$

In the case $2 \gamma_{0}=\gamma$ the proof follows from the theorem 3.3 .

\subsection{BEC.}

3.4.1. Coexistence of two non-conventional Bose-Einstein condensates. In this section we theoretically predict the exciting possibility of coexisting non-conventional Bose-Einstein condensates.

Theorem 3.5. Under the conditions $2 \gamma_{0}>\gamma$ and $\mu \geq \lambda\left(2 \gamma_{0}+\gamma\right) /\left(2 \gamma_{0}-\gamma\right)$, the system given by (6) displays simultaneous non-conventional Bose-Einstein condensation of the two levels associated to the zero mode and the amounts of condensate are given as:

$$
\rho_{\mathbf{0},-}(\mu)=\frac{\lambda}{2 \gamma_{0}-\gamma}+\frac{\mu}{2 \gamma_{0}+\gamma}
$$




$$
\rho_{\mathbf{0},+}(\mu)=-\frac{\lambda}{2 \gamma_{0}-\gamma}+\frac{\mu}{2 \gamma_{0}+\gamma}
$$

Proof. $p(\beta, \mu)$ can be rewritten as,

$$
p(\beta, \mu)=\frac{1}{4}\left(\frac{\left(\alpha_{-}-\alpha_{+}\right)^{2}}{2 \gamma_{0}-\gamma}+\frac{\left(\alpha_{-}+\alpha_{+}\right)^{2}}{2 \gamma_{0}+\gamma}\right)+p^{\mathrm{MF}^{\prime}}(\beta, \mu),
$$

where $\alpha_{-}=\lambda+\mu, \alpha_{+}=\mu-\lambda$. Using the convexity of $p_{l}(\beta, \mu)$ and $p_{l}^{\text {appr }}(\beta, c, \eta, \mu)$ respect to $\alpha_{-}$and $\alpha_{+}$we get from the Griffiths theorem [16] and theorem [3.3.

$$
\begin{gathered}
\rho_{\mathbf{0}, \sigma}=\lim _{V_{l} \rightarrow \infty} \partial_{\alpha_{\sigma}} p_{l}(\beta, \mu)=\partial_{\alpha_{\sigma}} \lim _{V_{l} \rightarrow \infty} p_{l}(\beta, \mu)=\partial_{\alpha_{\sigma}} \lim _{V_{l} \rightarrow \infty} \sup _{|c|,|\eta|: c, \eta \in \mathbb{C}} p_{l}(\beta, c, \eta, \mu) \\
=\partial_{\alpha_{\sigma}}\left(\frac{1}{4}\left(\frac{\left(\alpha_{-}-\alpha_{+}\right)^{2}}{2 \gamma_{0}-\gamma}+\frac{\left(\alpha_{-}+\alpha_{+}\right)^{2}}{2 \gamma_{0}+\gamma}\right)+p^{\mathrm{MF}^{\prime}}(\beta, \mu)\right)
\end{gathered}
$$

for $\sigma=-,+$. An analogous procedure leads to eq. (73) This yields to the proof of the theorem.

Corollary 3.6. For $\gamma=0$ we have,

$$
\rho_{\mathbf{0},-}=\left\{\begin{array}{ll}
0, & \mu \in(-\infty,-\lambda] \\
\frac{\mu+\lambda}{2 \gamma_{0}}, & \mu \in(-\lambda, \infty),
\end{array} \quad \rho_{\mathbf{0},+}= \begin{cases}0, & \mu \in(-\infty, \lambda] \\
\frac{\mu-\lambda}{2 \gamma_{0}}, & \mu \in(\lambda, \infty)\end{cases}\right.
$$

and for $\gamma=2 \gamma_{0}$,

$$
\rho_{\mathbf{0},-}= \begin{cases}0, & \mu \in(-\infty,-\lambda] \\ \frac{\mu+\lambda}{2 \gamma_{0}}, & \mu \in(-\lambda, \lambda], \\ \frac{\mu}{\gamma_{0}}, & \mu \in(\lambda, \infty],\end{cases}
$$

Proof. This proof is a direct consequence of Corollary 3.4 .

Let $\tau_{\mathrm{ss}, l}(\beta, \mu)$ and $\tau_{\mathrm{cs}, l}$ be thermal averages related to the self-scattering and crossscattering operators defined as:

$$
\tau_{\mathrm{ss}, l}(\beta, \mu)=V_{l}^{-1} \sum_{\sigma}\left\langle\left(\hat{a}_{\mathbf{0}, \sigma}^{\dagger}\right)^{2} \hat{a}_{\mathbf{0}, \sigma}^{2}\right\rangle_{\hat{H}_{l}(\mu)}, \quad \tau_{\mathrm{cs}, l}(\beta, \mu)=\left\langle\hat{\rho}_{\mathbf{0},+} \hat{\rho}_{\mathbf{0},-}\right\rangle_{\hat{H}_{l}(\mu)} .
$$

Corollary 3.7. For $2 \gamma_{0}>\gamma$ and $\mu \geq \lambda\left(2 \gamma_{0}+\gamma\right) /\left(2 \gamma_{0}-\gamma\right)$,

$$
\begin{gathered}
\lim _{V_{l} \rightarrow \infty} V_{l}^{-1} \tau_{\mathrm{cs}, l}(\beta, \mu)=\lim _{V_{l} \rightarrow \infty}\left\langle\hat{\rho}_{0,+} \hat{\rho}_{0,-}\right\rangle_{\hat{H}_{l}(\mu)}=\lim _{V_{l} \rightarrow \infty}\left\langle\hat{\rho}_{0,+}\right\rangle_{\hat{H}_{l}(\mu)}\left\langle\hat{\rho}_{0,-}\right\rangle_{\hat{H}_{l}(\mu)} \\
=\left(\frac{\mu}{2 \gamma_{0}+\gamma}\right)^{2}-\left(\frac{\lambda}{2 \gamma_{0}-\gamma}\right)^{2}
\end{gathered}
$$


Corollary 3.8. For $2 \gamma_{0}>\gamma>0, \mu \geq \lambda\left(2 \gamma_{0}+\gamma\right) /\left(2 \gamma_{0}-\gamma\right)$,

$$
\lim _{V_{l} \rightarrow \infty} V_{l}^{-1} \tau_{\mathrm{ss}, l}(\beta, \mu)=2\left(\left(\frac{\lambda}{2 \gamma_{0}-\gamma}\right)^{2}+\left(\frac{\mu}{2 \gamma_{0}+\gamma}\right)^{2}\right),
$$

and for $\gamma=0$,

$$
\lim _{V_{l} \rightarrow \infty} V_{l}^{-1} \tau_{\mathrm{ss}, l}(\beta, \mu)= \begin{cases}0, & \mu \in(-\infty,-\lambda] \\ \left(\frac{\mu+\lambda}{2 \gamma_{0}}\right)^{2}, & \mu \in(-\lambda, \lambda] \\ \frac{\mu^{2}+\lambda^{2}}{2 \gamma_{0}^{2}}, & \mu \in(\lambda, \infty) .\end{cases}
$$

Corollary 3.9. For $\gamma=2 \gamma_{0}$ the following identities

$$
\lim _{V_{l} \rightarrow \infty} V_{l}^{-1}\left(\tau_{\mathrm{ss}, l}(\beta, \mu)+2 \tau_{\mathrm{cs}, l}(\beta, \mu)\right)= \begin{cases}0, & \mu \in(-\infty,-\lambda] \\ \left(\frac{\mu+\lambda}{2 \gamma_{0}}\right)^{2}, & \mu \in(-\lambda, \lambda] \\ \frac{\lambda \mu}{\gamma_{0}^{2}}, & \mu \in(\lambda, \infty)\end{cases}
$$

hold.

\section{Generalized BEC}

We consider the case $g(x)=a x^{2}, a>0$. In this case Hamiltonians (6) and $\hat{H}_{l}^{\mathrm{MF}^{\prime}}(\mu)$ become

$$
\begin{gathered}
\hat{H}_{l}(\mu)=\sum_{\sigma, \mathbf{p} \in \Lambda_{l}^{*} \backslash\{0\}}\left(\left(\lambda_{l, \sigma}(\mathbf{p})-\mu_{l}\right) \hat{n}_{\mathbf{p}, \sigma}+\frac{\gamma_{0}}{V_{l}} \hat{n}_{\mathbf{p}, \sigma}^{2}\right)+\frac{a}{V_{l}}\left(\hat{N}^{\prime}\right)^{2}, \\
\hat{H}_{l}^{\mathrm{MF}^{\prime}}(\mu)=\sum_{\sigma, \mathbf{p} \in \Lambda_{l}^{*} \backslash\{0\}}\left(\lambda_{l, \sigma}(\mathbf{p})-\mu_{l}\right) \hat{n}_{\mathbf{p}, \sigma}+\frac{a}{V_{l}}\left(\hat{N}^{\prime}\right)^{2},
\end{gathered}
$$

respectively.

Let $p_{l}^{\prime}(\beta, \mu), p_{l}^{\mathrm{MF}^{\prime}}(\beta, \mu)$ be the grand canonical finite pressures associated to these operators. For every $\mu \in \mathbb{R}$, in the thermodynamic limit, it is proved in Ref.17 that

$$
\begin{aligned}
& \lim _{V_{l} \rightarrow \infty} p_{l}^{\prime}(\beta, \mu)=p^{\prime}(\beta, \mu)=\lim _{V_{l} \rightarrow \infty} p_{l}^{\mathrm{MF}^{\prime}}(\beta, \mu) \\
= & p^{\mathrm{MF}^{\prime}}(\beta, \mu)=\sup _{\alpha \leq 0}\left\{p^{\mathrm{id}}(\beta, \alpha)+\frac{(\mu-\alpha)^{2}}{2 a}\right\} .
\end{aligned}
$$


Proposition 4.1. Let

$$
\rho_{\mathrm{c}}^{\mathrm{id}}(\beta)=\frac{2}{(2 \pi)^{d}} \int_{[0, \infty)^{d}} \frac{d^{d} \mathbf{p}}{e^{\beta \frac{\|\mathbf{p}\|^{2}}{2}}-1}
$$

and $2 \gamma_{0}>\gamma$, then

$$
p(\beta, \mu)= \begin{cases}\frac{\lambda^{2}}{2 \gamma_{0}-\gamma}+\frac{\mu^{2}}{2 \gamma_{0}+\gamma}+p^{\mathrm{MF}^{\prime}}(\beta, \mu), & \frac{\lambda\left(2 \gamma_{0}+\gamma\right)}{2 \gamma_{0}-\gamma} \leq \mu \leq \frac{a}{2} \rho_{\mathrm{c}}^{\mathrm{id}}(\beta) \\ \frac{\lambda^{2}}{2 \gamma_{0}-\gamma}+\frac{\mu^{2}}{a}+\frac{\mu^{2}}{2 \gamma_{0}+\gamma}+p^{\mathrm{id}}(\beta, 0), & \mu \geq \frac{\lambda\left(2 \gamma_{0}+\gamma\right)}{2 \gamma_{0}-\gamma}>\frac{a}{2} \rho_{\mathrm{c}}^{\mathrm{id}}(\beta) .\end{cases}
$$

Moreover this model displays absence of macroscopic occupation of nonzero single levels:

$$
\lim _{V_{l} \rightarrow \infty}\left\langle\frac{\hat{n}_{\mathbf{p}, \sigma}}{V_{l}}\right\rangle_{\hat{H}_{l}(\mu)}=0
$$

for $\sigma=+,-$ and $\mathbf{p} \in \Lambda_{l}^{*} \backslash\{\mathbf{0}\}$, but generalized BEC can be verified in the sense that

$$
\lim _{\delta \rightarrow 0^{+}} \lim _{V_{l} \rightarrow \infty} \sum_{\sigma, \mathbf{p} \in \Lambda_{l}^{*}: \lambda_{l}(\mathbf{p}) \leq \delta}\left\langle\frac{\hat{n}_{\mathbf{p}, \sigma}}{V_{l}}\right\rangle_{\hat{H}_{l}(\mu)}>0,
$$

being $\hat{n}_{p}=\hat{n}_{\mathbf{p},-}+\hat{n}_{\mathbf{p},+}$.

\section{CONClusions}

The pressure of the model given by (6) can be exactly determined for a range of values of the chemical potential $\mu$ by using the energy operator (19) in the framework of the Bogolyubov approximation. The superstable system displays non-conventional BEC consisting in the macroscopic occupation of both ground state levels. The condensates can eventually coexist depending on the values of $\mu$. Moreover, as a subtle consequence of these results we have determined the exact values of some thermal averages associated to the cross scattering and self-scattering operators. Finally, in the case $g(x)=a x^{2}, a>0$, generalized BEC holds.

\section{ACKNOWLEDGEMENTS}

This work has been partially supported by Grant PBCT-ACT13 (Stochastic Analysis Laboratory, Chile), Programa de Magíster en Matemáticas, Universidad de La Serena and Program "Fundamental problems of nonlinear dynamics" of the RAS. 


\section{REFERENCES}

[1] A. Einstein, Sitzungsberichte der Preussischen Akademie der Wissenschaften I, 3 (1925).

[2] F. London, Phys. Rev. 54, 947 (1938).

[3] F. London, Superfluids. Vol. II (Wiley, New York, 1954).

[4] M.H. Anderson, J. R. Ensher, M.R. Mathews, C.E. Wieman, E. A. Cornell, Science 269, 198 (1995).

[5] C.C. Bradley, C. A. Sackett, J.J. Tollet, R.G. Hulet, Phys. Rev. Lett. 75, 1687 (1995).

[6] J.V. Pulé, A. F. Verbeure, V. A. Zagrebnov, J.Phys. A : Math. Gen. 37 (28), L231 (2004).

[7] J.V. Pulé, A. F. Verbeure, V. A. Zagrebnov, J.Phys. A : Math. Gen. 38 (23), 5173 (2005).

[8] N.N. Bogolyubov, Lectures on Quantum Statistics: Quasiaverages. Vol.2 (Gordon and Beach, New York, 1970).

[9] J. Ginibre, Commun. Math. Phys. 8, 26 (1968).

[10] M. van den Berg, J. T. Lewis, P. Smedt, J. Stat. Phys. 37 (5/6), 697 (1984).

[11] J.B. Bru, V.A. Zagrebnov, J.Phys. A : Math. Gen. 33, 449 (2000).

[12] J.B. Bru, V.A. Zagrebnov, Physica A268, 309 (1999).

[13] S. Choi, N. P. Bigelow, Phys. Rev. A. 72 (4), 033612 (2005).

[14] D. Ruelle, Statistical mechanics. Rigorous results ( W.A. Benjamin, New York, Amsterdam, 1969).

[15] A.V. Roberts, D.E.Varberg, Convex Functions (Academic Press, New York, 1973).

[16] R. Griffiths, J. Math. Phys. 5, 1215 (1964).

[17] T. Michoel, A.Verbeure, J. Math. Phys. 40 (3), 1268 (1999).

${ }^{1}$ Departamento de Matemáticas, Universidad de La Serena,Cisternas 1200, La Serena. Chile, mcorgini@yahoo.com

${ }^{2}$ Departamento de Matemáticas, Universidad de La Serena,Cisternas 1200, La Serena. Chile

${ }^{3}$ Steklov Mathematical Institute, Gubkin str. 8, 117966, Moscow, Russia, sankovch@mi.ras.ru 